\title{
Local Sequential Ensemble Kalman Filter for Simultaneously Tracking States and Parameters
}

\author{
Ning Zhou, Zhenyu Huang, Yulan Li \\ Energy and Environment Directorate \\ Pacific Northwest National Laboratory \\ Richland, WA 99352, USA \\ E-mail: \{ning.zhou, zhenyu.huang, yulan.li\}@pnnl.gov
}

\author{
Greg Welch \\ The Department of Computer Science \\ The University of North Carolina at Chapel Hill \\ Chapel Hill, NC 27599, USA \\ E-mail:welch@cs.unc.edu
}

\begin{abstract}
Accurate information about dynamic states and parameters is important for efficient control and operation of a power system. To improve the estimation accuracy of states and parameters, this paper applies a local sequential ensemble Kalman filter (EnKF) method to simultaneously estimate dynamic states and parameters using phasor-measurement-unit (PMU) data. Based on simulation studies using multi-machine systems, the proposed method performed favorably in tracking both states and parameters in real time.
\end{abstract}

\section{INTRODUCTION}

Electromechanical dynamic models are used widely to study problems involving transient and small signal stability in power systems. Accurate information about states (e.g., rotor speeds, angles) and parameters is essential for efficient control and operation of a power system. States are the minimum set of variables that can determine the current status of a dynamic system [1]. Parameters are the coefficients that relate the input, output, and state variables of a model. A dynamic model with accurate parameters and states can faithfully reveal system responses. Therefore, the model can be used to enhance stability control and establish accurate operation limits, which in turn improve the reliability and efficiency of the power system.

Traditionally, component-based approaches are used to build a dynamic model. The model parameters are derived from equipment manuals or staged tests [2]. A dynamic model can be used to answer 'what-if' questions regarding transient and small signal stability problems, provide dynamic security assessments for power system operations[3], and guide controller design. Yet, because of the large number of components in a typical power grid, efforts to build and maintain an accurate, comprehensive model are not trivial. This often results in a model that does not adequately reflect true system behaviors. For example, the initial model for simulating the U.S. Western Interconnection breakup on August 10, 1996 could not replicate the oscillations that were measured during the event [4]. The discrepancy between responses measured during a real event and responses from a simulation clearly reveals the inadequacy of the model. Low confidence in the accuracy of a model usually leads to conservative operation and reduces asset utilization. To improve model accuracy, many studies involving staged tests have been carried out to identify parameters $[5,6]$. However, most staged calibration methods require injecting probing signals and maneuvering real/reactive power, and therefore cannot capture parameter changes during normal operations. It would be of significant value if some parameters of dynamic models can be calibrated on line using event data.

The real-time states of electromechanical dynamics reflect the current status of a power system and can be used to coordinate controllers in a wide area. Traditionally, estimating dynamic states (e.g., rotor speeds, angles) over a wide area was not possible because data from the Supervisory Control and Data Acquisition (SCADA) system are normally sampled once every 2 to 4 seconds. This sampling rate is too low for the data to reveal electromechanical dynamic responses. In addition, SCADA data are not well synchronized. As a result, most controllers (e.g., power system stabilizers) for controlling the electromechanical dynamics only use local states to achieve local objectives. Compatibility among controllers is only studied in planning models during offline studies. Without global objectives and systematic coordination over wide areas, the influence of local controllers at the grid level may not always be desirable. For example, the wide area small signal problem has been associated with, “... the introduction of high gain, and low time constant automatic voltage regulators" [7].

The parameters and states of dynamic models can be estimated in real time using measurement data from phasor measurement units (PMU). These data typically have a sampling rate of 30 or 60 samples per second, are well synchronized with the Global Positioning System clock, and can continuously capture the dynamic responses of a power system under normal and abnormal conditions. Prior work by [8] revealed the benefits and potential of using PMU data with the extended Kalman filter (EKF) for online parameter identification and state estimation. Ghahremani et al. 
extended EKF to simultaneously estimate the generator states and unknown inputs [9], as well as using the unscented Kalman filter for estimating states using a single machine infinite bus system [10]. However, the required sampling rate of data in those studies are 2,000 samples per second and 10,000 samples per second, which is higher than what can be provided by a commercial PMU.

To overcome the difficulty, this paper proposes a local sequential ensemble Kalman filter (EnKF) method to simultaneously estimate dynamic states and parameters using PMU data. The paper is organized as follows. Section II formulates the state and parameter tracking problem using classical generator models and then generalizes the problem for other dynamic components. Section III provides an overview of the basic EnKF. Section IV presents a local sequential EnKF method. Section $V$ introduces the inflation method for the proposed EnKF. Section VI evaluates the performance of the proposed EnKF using simulation models. In Section VII, conclusions and future work are discussed.

\section{PRoBlem Formulation}

This section introduces the problem of tracking states and parameters using PMU data. First, a classical generator model is used as an example. The problem then is generalized using a non-linear dynamic model.

\section{A. Classical Generator Model}

A classical generator model can be described by (1) and (2) $[2]$ :

$$
\begin{gathered}
{\left[\begin{array}{c}
\dot{\delta} \\
\Delta \dot{\omega}
\end{array}\right]=\left[\begin{array}{cc}
0 & \omega_{0} \\
0 & -\frac{K_{D}}{2 H}
\end{array}\right]\left[\begin{array}{c}
\delta \\
\Delta \omega
\end{array}\right]+\left[\begin{array}{c}
0 \\
\frac{1}{2 H}
\end{array}\right]\left(T_{m}-T_{e}\right)} \\
T_{e}=P_{e}=\frac{E V}{X_{d}^{\prime}} \sin (\delta-\theta)
\end{gathered}
$$

where $\delta$ is the rotor angle and $\Delta \omega$ is the rotor speed deviation. The symbol $T_{m}$ is the mechanical torque, $T_{e}$ is the electric airgap torque, $H$ is the inertia constant, and $K_{D}$ is the damping factor. The symbol $\omega_{0}$ is the rated value of the rotor speed. The generator is connected to a terminal bus through a transient reactance $X_{d}^{\prime}$. The symbol $E$ is the internal voltage magnitude of the generator. Assume that PMU measurements of voltage and current phasors are available at the terminal bus of the generator. The state and parameter tracking problem for a classical generator model then can be stated as follows.

Using PMU measurements of voltages $(\widetilde{V}=V \angle \theta)$ and currents $(\tilde{I})$ at the generator buses, estimate the states of $\delta$, $\Delta \omega$, and model parameters of $H, K_{D}, X_{d}^{\prime}$.

Note that (1) and (2) are models only for a dynamic component (in this case, a generator) in a power grid. All the component models are coupled through the grid network model to form the full system model [2]. All the other variables (including $\widetilde{V}$ and $\tilde{I}$ ) can be derived from the states using the full system model.

\section{B. Generalized Dynamic Model}

To generalize the problem formulation, non-linear differential algebraic equations are used to describe power system dynamics as expressed in (3).

$$
\left\{\begin{array}{l}
\dot{x}=f_{c}(x, \alpha)+w_{c} \\
\dot{\alpha}=0+\varepsilon_{c} \\
z=h_{c}(x, \alpha)+v_{c}
\end{array}\right.
$$

where vector $x \in \mathrm{R}^{n x}$ represents state variables; vector $\alpha \in \mathrm{R}^{n \alpha}$ represents the parameters to be identified (for a classical generator; the parameters are $\left.H, K_{D}, X_{d}^{\prime}\right)$; vector $z \in \mathrm{R}^{m}$ represents available measurements (e.g., PMU measurements $\widetilde{V}$ and $\widetilde{I}$ at generator buses); the function $h(*)$ is the measurement function; the symbol " $c$ " indicates the continuous form of the model; and the random variables $w_{c} \in \mathrm{R}^{n x}, \varepsilon_{c} \in \mathrm{R}^{n \alpha}$, and $v_{c} \in \mathrm{R}^{m}$ represent process, parameter, and measurement noise, respectively. For a classical generator model, the states are $\delta$ and $\Delta \omega$ as shown in (1) and (2).

Assuming a sampling interval of $\Delta t$ seconds, the discrete form of the model corresponding to (3) can be written as (4).

$$
\left\{\begin{array}{l}
x_{k}=f\left(x_{k-1}, \alpha_{k-1}\right)+w_{k-1} \\
\alpha_{k}=\alpha_{k-1}+\varepsilon_{k-1} \\
z_{k}=h\left(x_{k}, \alpha_{k}\right)+v_{k}
\end{array}\right.
$$

where subscript " $k$ " denotes variables and quantities at time $k \Delta t$. The state transition function $f(*)$ in (4) can be implemented through numerical integration of $f_{c}\left(^{*}\right)$ in (3). The process, parameter, and measurement noise are assumed to be white noise with a mean value of zero and a covariance matrix of $Q$ and $R$ as defined by (5) and (6):

$$
\begin{gathered}
E\left(\left[\begin{array}{l}
w_{k} \\
\varepsilon_{k}
\end{array}\right]\left[\begin{array}{ll}
w_{k}^{T} & \varepsilon_{k}^{T}
\end{array}\right]\right)=Q \\
E\left(v_{k} v_{k}^{T}\right)=R
\end{gathered}
$$

The generalized state and parameter tracking problem then can then be stated as follows.

Using the PMU measurement sequence of $z_{1}, z_{2}, \ldots, z_{k}$, and model structure (4), (5), and (6), estimate the states of $x_{k}$ and the parameters of $\alpha_{k}$.

Note that unknown parameters $\alpha$ propagate over time in a way that is similar to the states $x$ in (4). By treating parameters as special states, and given sufficient measurements such that the system is observable, the state and parameter tracking problem can be solved using a Kalman filter.

\section{REVIEW OF AN ENSEMBLE KALMAN FILTER}

In this paper, EnKF is chosen for tracking states and parameters because it has been successfully applied in oceanographic studies (under the term "data assimilation") to track states of high-order nonlinear dynamic systems [11]. Compared to traditional EKF $[8,10]$, EnKF propagates the mean and covariance of states through ensembles by simulation. Therefore, users do not have to perform the linearization procedure required by EKF. A brief review of the 
notation and algorithm for a basic EnKF [11] are given as follows.

\section{A. Notation}

- Superscript $b$ is for background or prior states/ parameters. For example, $x_{k}^{b}$ denotes the estimated states at time step $k$ before the current measurement $z_{k}$ is used in the estimation.

- Superscript $a$ is for analysis or post state/parameters. For example, $x_{k}^{a}$ denotes the estimated states at time step $k$ after the current measurement $z_{k}$ is used in the estimation.

- $N$ is the total number of the ensembles (or samples).

- Superscript $(i)$ is the $i^{\text {th }}$ instance of the ensembles. Usually, $i=1,2, \cdots, N$.

\section{B. Algorithm of the Basic EnKF}

After initialization, the basic EnKF assimilates data one snapshot at a time. For one snapshot of data, there are two stages, i.e., a prediction stage and a correction stage. During the prediction stage, the states are propagated one time step ahead and form the background (or prior) states. During the correction stage, the analysis (or posterior) states are generated by correcting the background states with information from the latest measurements.

\section{1) Initialization}

Generate the ensembles for initial states and parameters to represent the probability distribution functions as follows:

$$
\begin{array}{cc}
x_{0}^{a(i)} \sim \operatorname{norm}\left(x_{0}, P_{x 0}\right) \in \mathrm{R}^{n x} & \text { for } i=1,2, \ldots, N ; \\
\alpha_{0}^{a(i)} \sim \operatorname{norm}\left(\alpha_{0}, P_{\alpha 0}\right) \in \mathrm{R}^{n \alpha} & \text { for } i=1,2, \ldots, N ;
\end{array}
$$

The norm term stands for normal distribution. MATLAB ${ }^{\mathbb{C}}$ function 'randn' is used to generate the ensembles for parameters and states that follows normal (or Gaussian) distributions.

\section{2) Prediction}

First, for all $i$, propagate the states to the next time step using (4) to form background states and parameters as follows:

$$
\left\{\begin{array}{l}
x_{k}^{b(i)}=f\left(x_{k-1}^{a(i)}, \alpha_{k-1}^{a(i)}\right)+w_{k-1}^{(i)} \\
\alpha_{k}^{b(i)}=\alpha_{k-1}^{a(i)}+\varepsilon_{k-1}^{(i)} \\
z_{k}^{b(i)}=h\left(x_{k}^{b(i)}, \alpha_{k}^{b(i)}\right)
\end{array}\right.
$$

where the ensembles $w_{k-1}^{(i)}$ and $\varepsilon_{k-1}^{(i)}$ are generated to represent the distribution of process and parameter noise as in (5).

Then, construct the Kalman gain $\left(K_{k}\right)$ using (8). The superscript "+" stands for the matrix pseudo-inversion operation. Note that neither measurement Jacobian matrix $H_{k}$ nor priori covariance matrix $P_{k}^{b}$ needs to be calculated explicitly. By applying the observation matrix-free implementation from section 4.2 of Mandel's publication [12], the terms in (8) can be estimated using (9), (10) and (11).

$$
\begin{aligned}
& K_{k}=\left(P_{k}^{b} H_{k}^{T}\right)\left(H_{k} P_{k}^{b} H_{k}^{T}+R\right)^{+} \\
& P_{k}^{b} H_{k}^{T} \approx \frac{1}{N-1}\left[\begin{array}{cccc}
\Delta x_{k}^{b(1)} & \Delta x_{k}^{b(2)} & \cdots & \Delta x_{k}^{b(N)} \\
\Delta \alpha_{k}^{b(1)} & \Delta \alpha_{k}^{b(2)} & \cdots & \Delta \alpha_{k}^{b(N)}
\end{array}\right]\left[\begin{array}{c}
\Delta z_{k}^{b(1)} \\
\Delta z_{k}^{b(2)} \\
\vdots \\
\Delta z_{k}^{b(N)}
\end{array}\right] \\
& H_{k} P_{k}^{b} H_{k}^{T} \approx \frac{1}{N-1}\left[\begin{array}{llll}
\Delta z_{k}^{b(1)} & \Delta z_{k}^{b(2)} & \cdots & \Delta z_{k}^{b(N)}
\end{array}\right]\left[\begin{array}{c}
\Delta z_{k}^{b(1)} \\
\Delta z_{k}^{b(2)} \\
\vdots \\
\Delta z_{k}^{b(N)}
\end{array}\right] \\
& R \approx \frac{1}{N-1} \sum_{i=1}^{N}\left(v_{k}^{(i)}\right)^{2}
\end{aligned}
$$

where ensembles $v_{k}^{(i)} \sim \operatorname{norm}(0, R) \in \mathrm{R}^{m}$ are generated to represent the distribution of measurement noise. To make estimates unbiased, make sure that $\sum_{i=1}^{N} v_{k}^{(i)}=0$ when $N$ is a finite number [13]. In addition, the state error ensembles $\left(\Delta x_{k}^{b(i)}\right)$ and parameter-error ensembles $\left(\Delta \alpha_{k}^{b(i)}\right)$ are defined by (12) and (13). The output error ensemble $\left(\Delta z_{k}^{b(i)}\right)$ is defined by (14), where $z_{k}^{b(i)} \stackrel{\Delta}{=} h\left(x_{k}^{b(i)}, \alpha_{k}^{b(i)}\right)$.

$$
\begin{gathered}
\Delta x_{k}^{b(i)} \stackrel{\Delta}{=} x_{k}^{b(i)}-\bar{x}_{k}^{b} \text { where } \bar{x}_{k}^{b}=\frac{\Delta}{N} \sum_{i=1}^{N} x_{k}^{b(i)} \\
\Delta \alpha_{k}^{b(i)} \stackrel{\Delta}{=} \alpha_{k}^{b(i)}-\bar{\alpha}_{k}^{b} \text { where } \bar{\alpha}_{k}^{b} \stackrel{\Delta}{=} \frac{1}{N} \sum_{i=1}^{N} \alpha_{k}^{b(i)} \\
\Delta z_{k}^{b(i) \stackrel{\Delta}{=}}=z_{k}^{b(i)}-\bar{z}_{k}^{b} \text { where } \bar{z}_{k}^{b} \stackrel{\Delta}{=} \frac{1}{N} \sum_{i=1}^{N} z_{k}^{b(i)}
\end{gathered}
$$

\section{3) Correction}

After a new set of measurements $\left(z_{k}\right)$ is obtained at time step $k$, the measurements are perturbed to generate the measurement ensembles $\left(z_{k}^{(i)}\right)$ as in (15). The information from measurements $z_{k}$ is included in the analysis results through (16). The mean of analysis ensembles $\left(\bar{x}_{k}^{a} \bar{\alpha}_{k}^{a}\right)$ defined by (17) is the estimate of the states and parameters by the basic EnKF method for time step $k$.

$$
\begin{gathered}
z_{k}^{(i)}=z_{k}+v_{k}^{(i)} \\
{\left[\begin{array}{c}
x_{k}^{a(i)} \\
\alpha_{k}^{a(i)}
\end{array}\right]=\left[\begin{array}{c}
x_{k}^{b(i)} \\
\alpha_{k}^{b(i)}
\end{array}\right]+K_{k}\left(z_{k}^{(i)}-z_{k}^{b(i)}\right)} \\
{\left[\begin{array}{c}
\bar{x}_{k}^{a} \\
\bar{\alpha}_{k}^{a}
\end{array}\right]=\frac{\Delta}{N} \sum_{i=1}^{N}\left[\begin{array}{l}
x_{k}^{a(i)} \\
\alpha_{k}^{a(i)}
\end{array}\right]}
\end{gathered}
$$

After the correction step, set $k=k+1$ and go to step 2. The prediction and correction procedure is repeated when a new measurement is available. It is known that the basic EnKF may suffer performance degradation problems [13]. To 
improve its applicability, extensions on the EnKF have been proposed. Two extensions adopted in this paper are described in the following sections.

\section{LOCAL SEQUENTIAL ENKF}

While the basic EnKF (described above in Section III) simultaneously assimilates all data in one snapshot, a sequential EnKF assimilates one subset of data at a time. To understand this concept, assume that the measurement data at step $k$ can be divided into $G$ subgroups, as shown in (18).

$$
z_{k}=\left[\begin{array}{llll}
z_{k, 1}^{T} & z_{k, 2}^{T} & \cdots & z_{k, G}^{T}
\end{array}\right]^{T} \in \mathrm{R}^{m}
$$

When the noise of the measurement data in different groups are uncorrelated, the data subset $z_{k, j}$ can be assimilated sequentially, one group at a time [14]. Assimilating data sequentially is a valid implementation of a Kalman filter as long as the measurements whose noise is correlated are assimilated in one block. The sequential EnKF can be programmed so that each subset of data can be processed as soon as they are available. There is no need to wait for all the data to arrive to start the correction procedure. Therefore, the sequential EnKF can be implemented with lower latency.

To improve estimation accuracy, it was proposed that a state be updated only with 'local' measurements [15], which leads to a local sequential EnKF. Local measurements refer to measurements that are highly correlated to the state of interest. These measurements are usually located close to the state; hence, they are named 'local' measurements.

There is noise in ensemble covariance matrixes, $P_{k}$, of the states. The noise usually leads to underestimation of prior covariance. Hamill and co-workers [15] showed that, when the noise levels in a covariance estimate is higher than the signal levels, assimilating corresponding data can degrade instead of improve the state (or parameter) estimate. In addition, [15] showed that estimation noise (or the variance of the estimates) increases when the magnitude of correlation is low. Therefore, to improve the accuracy of estimates, a local EnKF is used to reduce the influence of the observations (or measurements) that are weakly correlated to the states.

There are different approaches for implementing the local EnKF. In this paper, only the measurements taken at the generator terminal bus are used as local measurements to track states of the corresponding generator. Combined with the sequential EnKF described above, a local sequential EnKF can be implemented as follows. Assume that only a subset of states, $x_{k}\left(g_{x}\right)$ and $\alpha_{k}\left(g_{\alpha}\right)$, has a strong correlation with measurement group $z_{k, g}$. Here, $g_{x}$ and $g_{\alpha}$ are the index sequences that point to the states and parameters strongly correlated to measurement group $g$. Assume that the correlation between $z_{k, g}$ and the remaining states and parameters is weak. Therefore, the correlation is considered to be zero to avoid degradation resulting from estimation errors. Then, the local sequential EnKF can be implemented by replacing (8) through (16) with the following steps and (19) through (25):

\section{1) Initialize the analysis step}

$$
\left[\begin{array}{c}
x_{k, 0}^{b(i)} \\
\alpha_{k, 0}^{b(i)}
\end{array}\right]=\left[\begin{array}{c}
x_{k}^{b(i)} \\
\alpha_{k}^{b(i)}
\end{array}\right] \text { and } g=0 ;
$$

2) $g=g+1$

3) Construct the Kalman gain

$$
\begin{gathered}
K_{k, g}\left(g_{x}, g_{\alpha}\right)=\left(P_{k}^{b}\left(g_{x}, g_{\alpha}\right) H_{k, g}^{T}\right)\left(H_{k, g} P_{k}^{b} H_{k, g}^{T}+R_{k, g}\right)^{+} \\
P_{k}^{b} H_{k, g}^{T} \approx \frac{1}{N-1}\left[\begin{array}{ccc}
\Delta x_{k}^{b(1)}\left(g_{x}\right) & \cdots & \Delta x_{k}^{b(N)}\left(g_{x}\right) \\
\Delta \alpha_{k}^{b(1)}\left(g_{\alpha}\right) & \cdots & \Delta \alpha_{k}^{b(N)}\left(g_{\alpha}\right)
\end{array}\right]\left[\begin{array}{c}
\Delta z_{k, g}^{b(1)} \\
\Delta z_{k, g}^{b(2)} \\
\vdots \\
\Delta z_{k, g}^{b(N)}
\end{array}\right] \\
H_{k, g} P_{k}^{b} H_{k, g}^{T} \approx \frac{1}{N-1}\left[\begin{array}{llll}
\Delta z_{k, g}^{b(1)} & \Delta z_{k, g}^{b(2)} & \cdots & \Delta z_{k, g}^{b(N)}
\end{array}\right]\left[\begin{array}{c}
\Delta z_{k, g}^{b(1)} \\
\Delta z_{k, g}^{b(2)} \\
\vdots \\
\Delta z_{k, g}^{b(N)}
\end{array}\right]
\end{gathered}
$$

4) Perform the observation perturbation

$$
z_{k, g}^{(i)}=z_{k, g}+v_{k, g}^{(i)}
$$

5) Update the states and parameters according to (24)

$$
\left[\begin{array}{l}
x_{k, g}^{b(i)}\left(g_{x}\right) \\
\alpha_{k, g}^{b i(}\left(g_{\alpha}\right)
\end{array}\right]=\left[\begin{array}{l}
x_{k, g-1}^{b(i)}\left(g_{x}\right) \\
\alpha_{k, g-1}^{b(i)}\left(g_{\alpha}\right)
\end{array}\right]+K_{k}\left(g_{x}, g_{\alpha}\right)\left(z_{k, g}^{(i)}-z_{k, g}^{b(i)}\right)
$$

6) If $g<G$, go to step 1); otherwise, go to step 7).

7) When $g=G$ (i.e., all the measurement subsets have been processed for the correction step), assign

$$
\left[\begin{array}{c}
x_{k}^{a(i)} \\
\alpha_{k}^{a(i)}
\end{array}\right]=\left[\begin{array}{c}
x_{k, G}^{b(i)} \\
\alpha_{k, G}^{b(i)}
\end{array}\right]
$$

and proceed to the prediction step for next time instance $(k=k+1)$.

\section{INFLATION METHOD}

In addition to the localization method, an inflation approach has been used to deal with underestimation of the covariance of background states [13]. Because of sampling errors associated with EnKF in calculating the Kalman gain, the estimated covariance matrix can become smaller than the true covariance. This, in turn, results in overweighting background states over new observations, and may eventually lead to the divergence of EnKF. An inflation method is implemented to increase the covariance of ensembles to counteract the overweighting on the background states by adding inflation equations (i.e., (26) and (27)) after (24).

$$
\begin{gathered}
x_{k, g}^{b(i)}\left(g_{x}\right)=\sqrt{\gamma_{x}}\left(x_{k, g}^{b(i)}\left(g_{x}\right)-\bar{x}_{k, g}^{b}\left(g_{x}\right)\right)+\bar{x}_{k, g}^{b}\left(g_{x}\right) \\
\alpha_{k, g}^{b(i)}\left(g_{\alpha}\right)=\sqrt{\gamma_{\alpha}}\left(\alpha_{k, g}^{b(i)}\left(g_{\alpha}\right)-\bar{\alpha}_{k, g}^{b}\left(g_{\alpha}\right)\right)+\bar{\alpha}_{k, g}^{b}\left(g_{\alpha}\right)
\end{gathered}
$$

where $\bar{x}_{k, g}^{b}\left(g_{x}\right)=\frac{1}{N} \sum_{i=1}^{N} x_{k, g}^{b(i)}\left(g_{x}\right)$ and $\bar{\alpha}_{k, g}^{b}\left(g_{x}\right)=\frac{1}{N} \sum_{i=1}^{N} \alpha_{k, g}^{b(i)}\left(g_{x}\right)$. Here, $\sqrt{\gamma_{x}}$ and $\sqrt{\gamma_{\alpha}}$ are inflation factors with values slightly larger than 1. For simplicity, this paper uses a fixed inflation factor of 1.01 as suggested by [13]. 


\section{CASE STUdiES}

Simulation studies are carried out to evaluate the performance of the proposed local sequential EnKF. An IEEE 3-machine 9-bus model (Fig. 1) [16] is used illustrate the applicability of the proposed method. All the generators were modeled using the classical model as described by (1) and (2). Using the Power System Toolbox (PST) [17], dynamic responses are generated by simulating a three-phase-to-ground fault with all correct parameters. The fault is applied at bus 6 at 1.0 second and is cleared in 0.05 seconds. The simulation is performed at a sampling rate of $1000 \mathrm{samples} / \mathrm{sec}$.

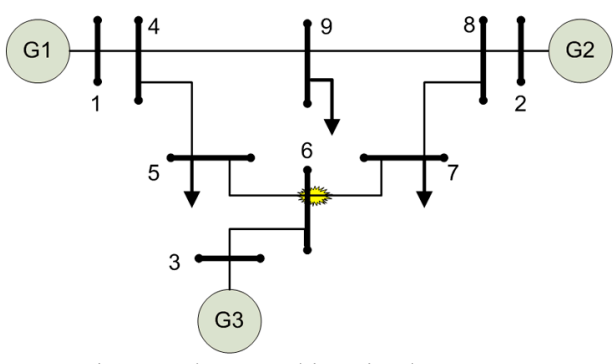

Fig. 1. A three-machine nine-bus system.

Assume that PMUs are available at all the generator buses to measure the voltage and the current phasors at a sampling rate of 25 samples/s. PMU measurements are generated by adding noise to the simulated responses. According to IEEE Standard C37.118-2005 [18], PMU measurement errors shall be lower than $1 \%$ of total vector errors. Accordingly, $1 \%$ of the total-vector-error white noise is added to the dynamic responses to simulate PMU measurements. The PMU measurements at each generation bus are converted into bus voltage magnitudes, bus voltage angles, real powers, and reactive powers from the generators. Measurements at generation bus " $g$ " are grouped into one data subset $z_{k, g}$ and are treated as local measurements for the corresponding generator's states and parameters.

Assume parameter errors exist in generator models. To simulate the modeling errors, the parameters of generators (e.g., inertia $H$, reactance $X_{d}^{\prime}$, and damping coefficient $K_{D}$ ) are perturbed, and the resulting perturbed parameters are summarized in Table I and used as initial values in EnKF. The true parameters and states are used as a yardstick to evaluate the tracking accuracy and speed.

The local sequential EnKF is set up to track the dynamic states $(\delta, \Delta \omega)$ and parameters $\left(H, K_{D}, X_{d}^{\prime}\right)$ of classical generator models. The ensemble size $N$ is set to be 75 . Both the state-transition and measurement functions in the prediction step of (7) can be implemented through the simulation functions available in the PST. The second-order Euler method is used for the numerical integration. To reduce errors, the integration time step is selected to be $10 \mathrm{~ms}$. Since the PMU sampling interval (i.e., $\Delta t$ ) is $40 \mathrm{~ms}$, (3) needs to be integrated over four time steps for each prediction step.

The parameter tracking results from the basic EnKF and the proposed local sequential EnKF are compared, using relative errors, in Fig. 2, 3, and 4. Relative error of $0 \%$ indicates perfect tracking. The state tracking results for generator 1 are compared in Fig. 5.

TABle I. PERTURbed VALUES OF THE GENERATOR PARAMETERS

\begin{tabular}{c|c|c|c|c|c|c}
\hline & \multicolumn{2}{|c|}{ H } & \multicolumn{2}{c|}{$X_{d}^{\prime}$} & \multicolumn{2}{c}{$\mathbf{K}_{\mathbf{D}}$} \\
\hline $\mathbf{G 1}$ & 16.37 & $+20 \%{ }^{*}$ & 0.0730 & $+20 \%{ }^{*}$ & 14.40 & $+50 \%{ }^{*}$ \\
\hline $\mathbf{G 2}$ & 7.68 & $+20 \%{ }^{*}$ & 0.0958 & $-20 \%{ }^{*}$ & 1.25 & $-50 \%{ }^{*}$ \\
\hline $\mathbf{G 3}$ & 2.41 & $-20 \%{ }^{*}$ & 0.2176 & $+20 \%{ }^{*}$ & 1.50 & $-50 \%{ }^{*}$ \\
\hline$*$ The relative errors of the perturbed parameters with respect to the true
\end{tabular}
values.

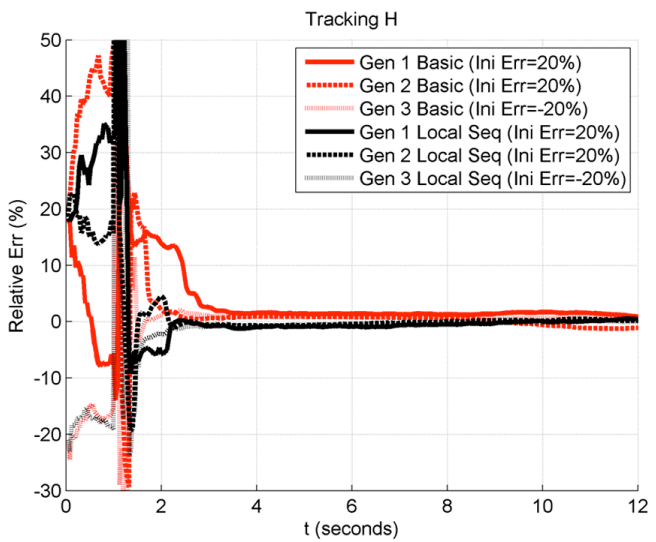

Fig. 2. Relative errors of estimated inertia (H)

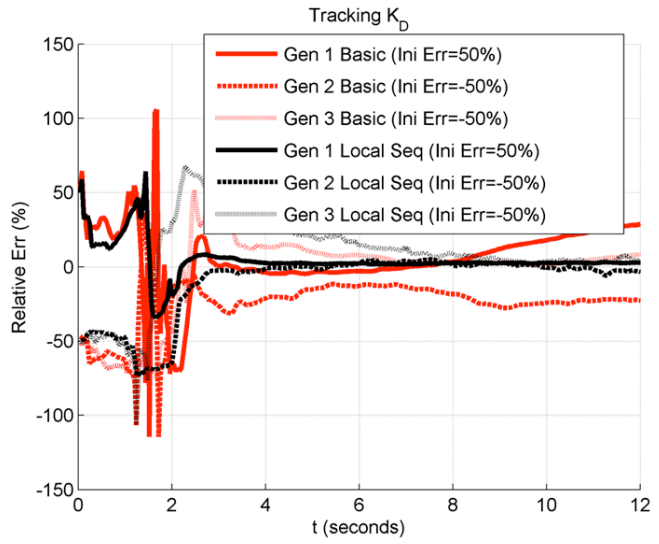

Fig. 3. Relative errors of estimated damping factor $\left(K_{D}\right)$

As can be seen from the figures, all the parameters converge well towards their true value for both methods, but the local sequential EnKF method exhibits better overall tracking accuracy and faster convergence. For inertia $(H)$ in Fig. 2, the local sequential EnKF has slightly better accuracy and much faster convergence. For the damping coefficient $\left(K_{D}\right)$ in Fig. 3, the local sequential EnKF has much better accuracy, while the basic EnKF has errors that stay around $25 \%$. For the transient reactance $\left(X_{d}^{\prime}\right)$ in Fig. 4, the convergence rates of the two methods appear to be similar.

Note that the values of $X_{d}^{\prime}$ are tracked during steady-state responses. As it can be seen from (2), $X_{d}^{\prime}$ influences the steady-state responses and is therefore identifiable during the steady-state responses. In contrast, $H$ and $K_{D}$ do not track the true values during the steady-state responses. They only converge to true values during the transient responses. This is due to $H$ and $K_{D}$ only influencing the transient responses in 
(1). Changing $H$ and $K_{D}$ does not change the steady-state responses. Also note that $H$ can be tracked relatively faster than $K_{D}$. This observation reveals that the tracking capability of EnKF is different for different parameters for the same measured data.

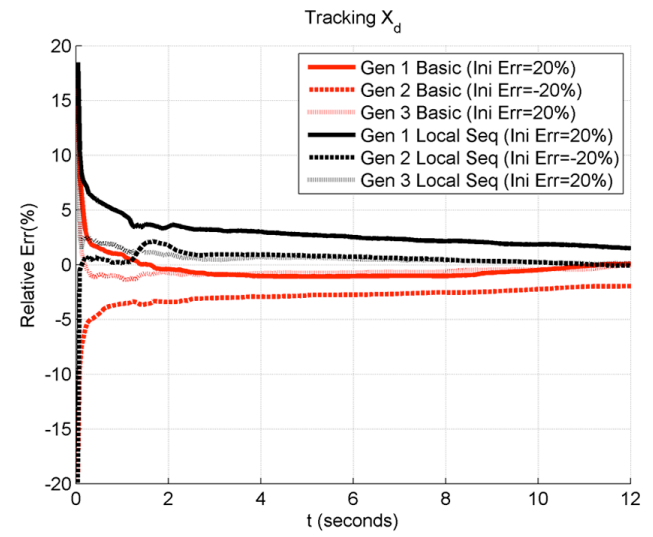

Fig. 4. Relative errors of estimated transient reactance $\left(X_{d}^{\prime}\right)$
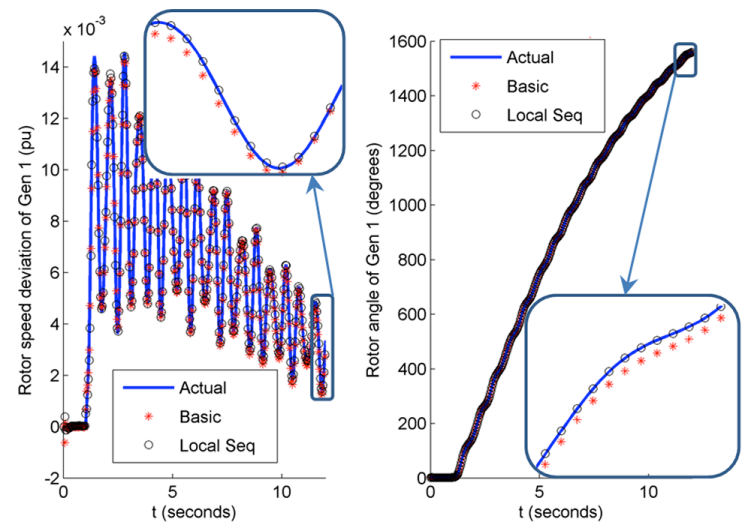

Fig. 5. States tracking results for generator 1

As seen in Fig. 5, both methods can track the rotor speed deviation $\left(\Delta \omega_{1}\right)$ and rotor angle $\left(\delta_{l}\right)$ of generator 1 very well. The mean absolute errors (MAE) of the estimated states for all the generators are summarized in Table II. As can be observed from the data, the local sequential EnKF yields much smaller MAEs than the basic EnKF, which indicates that the local sequential EnKF performs better than the basic EnKF.

TABLE II. MAEs OF THE ESTIMATED ROTOR ANGLE ( $\delta$ IN DEGRESS) AND $\operatorname{SPEED}$ DEVIATION $(\Delta \omega$ in $\mathrm{pu})$

\begin{tabular}{c|ccc|ccc}
\hline MAE & $\delta_{1}$ & $\delta_{2}$ & $\delta_{3}$ & $\Delta \omega_{1}$ & $\Delta \omega_{2}$ & $\Delta \omega_{3}$ \\
\hline $\begin{array}{c}\text { Basic } \\
\text { Local } \\
\text { Sequential }\end{array}$ & 0.80 & 1.03 & 0.84 & $1.3 \times 10^{-4}$ & $1.3 \times 10^{-4}$ & $1.9 \times 10^{-4}$ \\
\hline
\end{tabular}

\section{CONCLUSIONS AND FUTURE WORK}

The local sequential EnKF method is successfully formulated and applied to simultaneously track the states and parameters of power system dynamic models using PMU measurements. The proposed EnKF method is non-intrusive simulation based approach in that it propagates the mean and covariance of states through ensembles by simulation The performance of the proposed method is validated through simulation studies on classical generator models. Testing under realistic conditions of phasor data speed, measurement noise, and parameter errors in the simulation studies demonstrates the applicability of the proposed method for real-world problems.

\section{REFERENCE}

[1] P. DeRusso, R. Roy, C. Close and A.A. Desrochers, State Variables for Engineers. New York: Wiley, $2^{\text {nd }}$ edition, 1998.

[2] P. Kundur, Power System Stability and Control, New York: McGrawHill, Inc., 1994.

[3] A. A. Fouad, F. Aboytes, V. F.Carvalho, S. L. Corey; K. J. Dhir, R. Vierra, "Dynamic security assessment practices in North America," IEEE Transactions on Power Systems, vol.3, no. 3, pp. 1310-1321, August 1988.

[4] D. N. Kosterev, C. W. Taylor, and W. A. Mittelstadt, "Model validation for the August 10,1996 WSCC system outage," IEEE Transactions on Power Systems, vol. 14, no. 3, pp. 967-979, August 1999.

[5] P. Pourbeik, and F. Modau, "Model development and field testing of a heavy-duty gas-turbine generator," IEEE Transactions on Power Systems, vol. 23, no. 2, pp. 664-672, May 2008.

[6] J. Chow, M. Glinkowski, R. Murphy, T. W. Cease, and N. Kosaka, "Generator and exciter parameter estimation of Fort Patrick Henry hydro unit 1," IEEE Transactions on Energy Conversion, vol. 14, no. 4, pp. 923-929, Dec. 1999,.

[7] M. A. Pai, D. P. Sen Gupta and K. R. Padiyar, Small Signal Analysis of Power Systems, Harrow: Alpha Science International, 2004.

[8] Z. Huang, P. Du, D. Kosterev, B. Yang, "Application of extended Kalman filter techniques for dynamic model parameter calibration," IEEE Power Engineering Society General Meeting, Calgary, Canada, pp. 1-8, July 2009.

[9] E. Ghahremani, I. Kamwa, "Dynamic State Estimation in Power System by Applying the Extended Kalman Filter With Unknown Inputs to Phasor Measurements," IEEE Transactions on Power Systems, vol. 26, no. 4, pp 2556-2566, Nov 2011.

[10] E. Ghahremani, I. Kamwa, "Online State Estimation of a Synchronous Generator Using Unscented Kalman Filter From Phasor Measurements Units," IEEE Transactions on Energy Conversion, vol 26, no.4, 1099 1108, Dec 2011.

[11] G. Evensen, "Sequential data assimilation with a nonlinear quasigeostrophic model using Monte-Carlo methods to forecast error statistics," Journal of Geophysical Research, vol. 99, no. C5, pp. 14310 162. May 1994.

[12] J. Mandel, A Brief Tutorial on the Ensemble Kalman Filter, Center for Computational Mathematics Reports No 242, University of Colorado at Denver, 2007.

[13] G. Evensen, "The ensemble Kalman filter for combined state and parameter estimation: Monte Carlo techniques for data assimilation in large systems," IEEE Control System Magazine, vol. 29, no. 3, pp. 83104, 2009.

[14] G. Welch, and G. Bishop, "SCAAT: Incremental tracking with incomplete information," Computer Graphics, 31(Annual Conference Series), pp. 333-344, 1997.

[15] T. M. Hamill, J. S. Whitaker, C. Snyder, "Distance-dependent filtering of background error covariance estimates in an ensemble Kalman filter," Monthly Weather Review, vol. 129, pp. 2776-2790, 2001.

[16] P. M. Anderson and A. A. Fouad, Power System Control and Stability, Ames, IA, USA: Iowa State Univ. Press, 1977.

[17] J. H. Chow; and KW Cheung, "A toolbox for power system dynamics and control engineering education and research," IEEE Transactions on Power Systems, vol.7, no.4, pp.1559-1564, Nov. 1992.

[18] K. E. Martin, D. Hamai, et. al., "Exploring the IEEE standard C37.1182005 synchrophasors for power systems", IEEE Transactions on Power Delivery, vol. 23, no. 4, pp. 1805-1811, Oct. 2008. 